\title{
The VERA project
}

\author{
Mareki Honma \\ VERA Project office, National Astronomical Observatory of Japan, \\ 181-8588 Mitaka, Japan
}

\begin{abstract}
I present an overview of the VERA (VLBI Exploration of Radio Astrometry) project. I summarize the properties of the VERA array and its observational system as well as the current construction status, and I also present a brief overview of the science targets of VERA, including dynamics of the Galaxy and internal kinematics of maser sources.
\end{abstract}

\section{Introduction}

VERA (VLBI Exploration of Radio Astrometry) is a new VLBI array in Japan, being promoted by National Astronomical Observatory of Japan under collaboration with several Japanese universities (Sasao 1996; Honma et al. 2000a). The VERA array is designed for phase-referencing VLBI, with its antennas having dual-beam system which enables us to observe a Galactic maser source and a phase calibrator (reference) source simultaneously (Kawaguchi et al. 2000). With this system, VERA will measure positions of Galactic maser sources relative to reference sources (QSOs and radio galaxies) with 10 microarcsec level accuracy. Hence, VERA will be one of most powerful tools to study the dynamics of the Milky Way as well as internal kinematics of maser sources. In this paper, we present a brief overview of the VERA project including system construction as well as science targets.

\section{System Overview}

The array of VERA consists of four 20-m antennas spread over Japan (see figure 1 for the location of VERA stations). The latitudes of VERA stations varies from $24^{\circ}$ to $39^{\circ}$, and the baseline length ranges from $1200 \mathrm{~km}$ to $2300 \mathrm{~km}$. Three of the four stations are operational since April 2001, and the fourth station (in Ishigaki island) will be commissioned by April 2002. Figure 2 is a view of Mizusawa station (as of April 2001) showing the 20-m VERA's antenna (on the right $10 \mathrm{~m}$ antenna is also seen). VERA's antenna is designed for observational frequency from $2 \mathrm{GHz}$ to $43 \mathrm{GHz}$ and may be usable up to $86 \mathrm{GHz}$. For phasereferencing observation, each antenna is installed with dual-beam system on which two receivers are mounted. The dual-beam system is fully steerable, and can be as large as 2 degrees apart, with a minimum separation of 0.5 degree (see figure 3 for the dual-beam platform). Receivers are mounted on the platforms sustained by six arms, and its position is shifted by changing the lengths of each arm. 


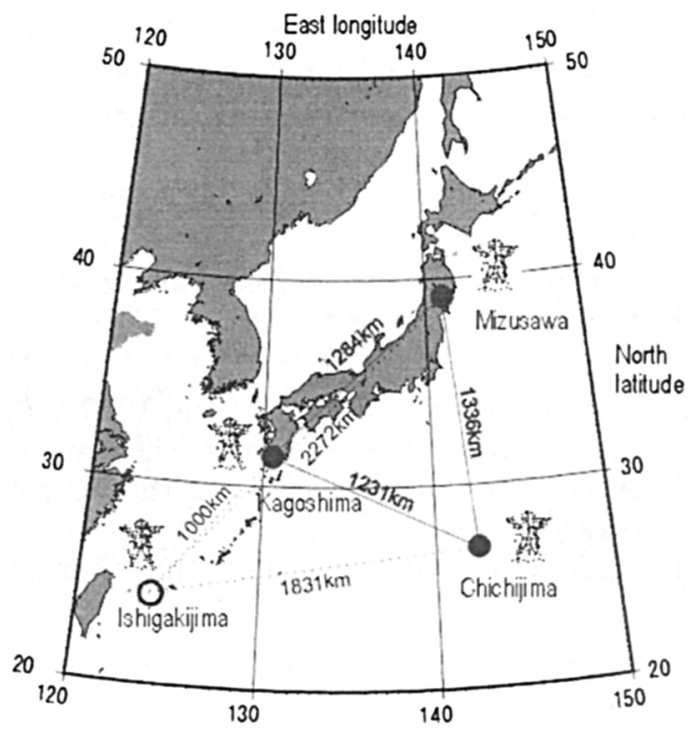

Figure 1. VERA array map. Filled circles are stations constructed by 2001 April. The Ishigaki-jima station (open circle) is to be constructed by 2002 April.

Receiver system for VERA includes following four bands; $\mathrm{S}$ band (2 GHz), $\mathrm{X}$ Band $(8 \mathrm{GHz}), \mathrm{K}$ band $(22 \mathrm{GHz})$ and $\mathrm{Q}$ band $(43 \mathrm{GHz})$. The $\mathrm{S}$ and $\mathrm{X}$ band receivers are mainly for geodetic observations to determine baseline parameters, and only one set of $\mathrm{S}$ and $\mathrm{X}$ band receivers is installed to each station. The receivers for the $\mathrm{K}$ and $\mathrm{Q}$ bands are for $\mathrm{H}_{2} \mathrm{O}$ and $\mathrm{SiO}$ masers as well as continuum sources to be used as a phase reference, and two pairs of $\mathrm{K}$ and $\mathrm{Q}$ band receivers are installed at each station. All of VERA's receivers are equipped with HEMT amplifiers, and the noise temperature for $\mathrm{K}$ band receiver is expected to be less than $60 \mathrm{~K}$, and for $\mathrm{Q}$ band receiver around $100 \mathrm{~K}$.

The receiver output signals are converted to an intermediate frequency (IF) of 5 to $7 \mathrm{GHz}$, and after a baseband downconversion, it undergoes the digital processing. For digital recording system, we have developed an ultra-high-speed digital recorder which works at the maximum rate of 1 gigabits per second. This high rate provide us a total bandwidth of $256 \mathrm{MHz}$ with a 2-bit signal quantization. Digitized data will be stored on magnetic tapes, and will be sent to the correlation center located at the Mitaka campus of NAOJ. The data will be processed with the NAOJ FX correlator, which was originally developed for VLBI Space Observatory Program (VSOP), and has been improved for VERA, in particular for high speed recording and dual-beam phase-referencing astrometry.

Since the radio waves from two sources (source and reference) go through completely different paths in the receiving system, phase differences due to instrumental delays produces an error in phase measurement. In order to correct for this instrumental delay, a noise source is mounted on the main reflector of antenna and a signal tune is injected into dual-beam receivers after being fo- 

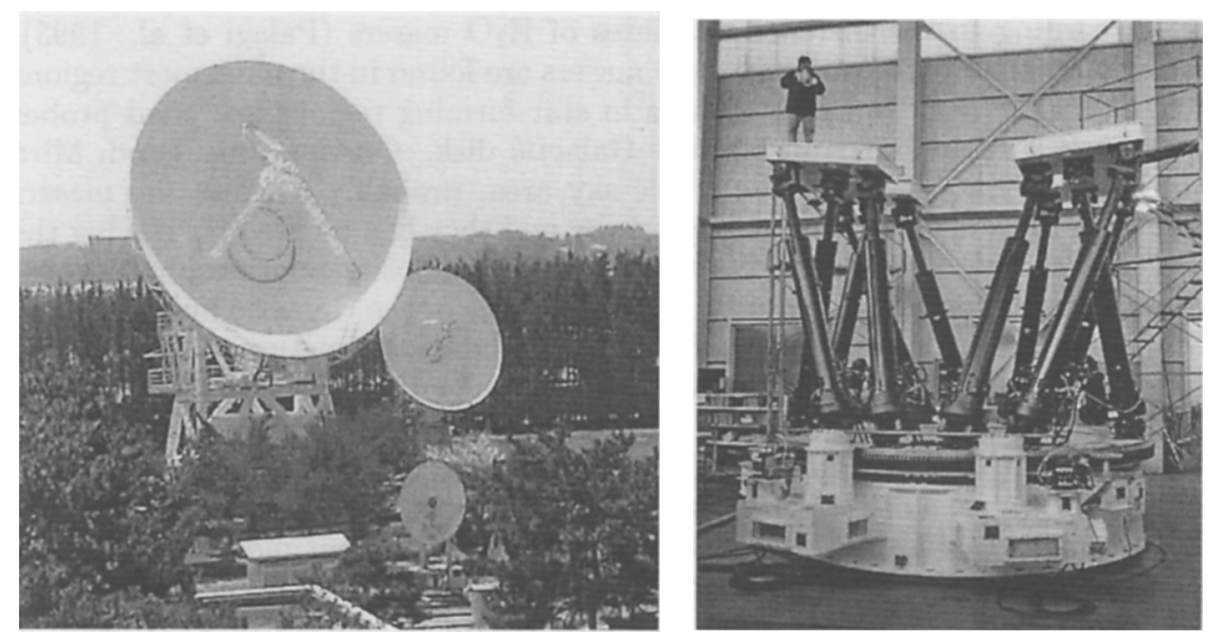

Figure 2. (left) View of VERA Mizusawa station. VLBI 10m antenna is also seen on the right to the VERA antenna.

Figure 3. (right) Dual-beam platform system.

cussing by the subreflector. The correlation of this noise source (observed with dual-beam receivers) is being monitored on real-time to obtain the instrumental delay and its time-variation. A real-time correlation system is installed at each station and it correlates the outputs of dual-beam receivers at each station. This correlation does not produce any fringe of astronomical objects, since dual-beam receivers observe two independent sources which are completely incoherent. With such a calibration system, one will be able to calibrate the instrumental delay within $0.05 \mathrm{~mm}$, and the relative delay of both target and reference sources within $0.1 \mathrm{~mm}$, including the residual atmospheric fluctuations. Once the relative delay is obtained within $0.1 \mathrm{~mm}$, the position accuracy will be $\sim 10 \mu \operatorname{arcsec}(=0.1 \mathrm{~mm} / 2300 \mathrm{~km}$, where $2300 \mathrm{~km}$ is the baseline length).

\section{Science Overview}

VERA aims to measure positions of Galactic maser sources relative to reference sources (QSOs and radio galaxies) with around 10 microarcsec level positional accuracy. This accuracy allows us to determine the distance of an object D $\mathrm{kpc}$ away with uncertainty of $\mathrm{D} \%$, and the proper motion with uncertainty of $0.05 \mathrm{D} \mathrm{km} / \mathrm{s}$. Therefore, VERA will be able to determine parallaxes and proper motions of maser sources in the whole Galaxy.

The main targets to be observed with VERA are Galactic $\mathrm{H}_{2} \mathrm{O}(22 \mathrm{GHz})$ and $\mathrm{SiO}(43 \mathrm{GHz})$ masers that are mainly emitted from star forming regions and Mira-type variables. $\mathrm{H}_{2} \mathrm{O}$ masers from star forming regions are much brighter than those of Mira-type stars, and are observable in the entire Galactic disk. In 
fact, according to the distance estimates of $\mathrm{H}_{2} \mathrm{O}$ masers (Palagi et al. 1993), several star forming regions with $\mathrm{H}_{2} \mathrm{O}$ masers are found in the outermost regions of the Galactic disk and $\mathrm{H}_{2} \mathrm{O}$ masers in star forming regions are good probes of the structure and dynamics of the Galactic disk. On the other hand, Mira variables are scattered over the whole sky area, probably because the masers from Mira-type variables are mostly nearby and they can be used for tracing the local disk as well as for studying their period-luminosity relationships. There are also a fairly large number of $\mathrm{SiO}$ maser sources in the Galactic bulge. Hence, Mira-type stars are not only useful for studying the local structure of the Galactic disk, but also useful for studying the Galactic bulge itself.

In addition to maser sources, VERA can also observe continuum sources that are bright at $22 \mathrm{GHz}$ and $43 \mathrm{GHz}$ bands, mainly as phase references. Currently, a few thousands of VLBI sources (compact sources detectable with VLBI) are known (e.g., Ma et al. 1998; Peck \& Beasley 1998), but most of surveys of VLBI sources have been performed for $|b| \geq 2.5^{\circ}$. As a first step to search for VLBI sources in the Galactic plane, we have conducted survey of VLBI sources in the Galactic plane using Japanese VLBI network, finding more than 50 new VLBI sources (Honma et al. 2000b). After that survey, more than $400 \mathrm{H}_{2} \mathrm{O}$ masers have a reference source within $2^{\circ}$.

One of the major scientific targets of VERA is of course to investigate the dynamics of the Galaxy. This includes: I) determination of the distance to the Galaxy center based on the parallax of Sgr A, II) determination of the Galactic rotation velocity at the Sun $\left(\Theta_{0}\right)$ by measuring motions of local maser sources, III) measurement of outer rotation curve based on the distance and three-dimensional velocities of outer maser sources, IV) revealing the shape of the Galaxy traced by maser sources in the Galactic bulge, disk, and spiral arms, and so on.

Also, VERA will provide a new possibility to study the internal kinematics in maser sources based on an absolute reference, which will be of great use for investigating the kinematics of star forming regions and physics of circumstellar regions in Mira variables.

\section{References}

Honma, M., et al. 2000a, in Radio Telescope, ed. H. R. Buthcer, Proc. SPIE 4015,624

Honma, M., et al. 2000b, PASJ, 52, 631

Kawaguchi, N., Sasao, T., \& Manabe, S. 2000, in Radio Telescope, ed. H. R. Butcher, Proc. SPIE 4015, 544

Ma C., Arias, E.F., Eubanks T.M., Fey, A.L., Gontier, A.M., Jacobs, C.S., Sovers, O.J., Archnial, B.A., Charlot, P. 1998, AJ, 118, 516

Palagi, F., Cesaroni, R., Comoretto, G., Felli, M. \& Natale, V. 1993, A\&A, 101, 153

Peck, A.B., Beasley A.J., in ASP Conference Series Vol.144, IAU Colloquium 464, eds, J.A. Zensus, G.B. Taylor, and J.M. Wrobel, pp.155

Sasao, T. 1996, in Proc. of 4th Asia-Pacific Telescope Workshop, ed. E. A. King, 94 (Sydney:Australia Telescope National Facility) 